\title{
WAVE MOTION IN AN ELASTIC SOLID DUE TO A NONUNIFORMLY MOVING LINE LOAD*
}

\author{
BY \\ L. B. FREUND \\ Brown University
}

\begin{abstract}
The wave motion resulting from a line load moving through an unbounded elastic solid is considered. The line load suddenly appears in the body, and then moves in a fixed direction with nonuniform speed. Exact expressions are derived for the displacement potential functions for all places in the body, for all time, by Laplace transform methods. The wavefronts, particularly the Mach waves trailing the moving load, are determined by integrating the bicharacteristic equations. The details of the wavefront shapes are illustrated and discussed for a load accelerating or decelerating in a prescribed way. Finally, a sample first-motion calculation is carried out in which the mean normal stress is determined immediately behind the dilatational wavefront by asymptotic methods.
\end{abstract}

Introduction. The problem of determining the response of an elastic solid to moving loads has been of interest for some time. The motivation for studying problems of this kind has been provided by the fields of structural dynamics, seismology, and related areas. The particular moving-load problems which have been studied may be put into one of three categories, according to the way in which the position of the load depends on time. The three categories are: (i) steady wave motion due to a load moving with constant velocity for all time, (ii) transient wave motion due to a load which begins to act at a certain instant and then moves with constant velocity, and (iii) transient wave motion due to a load which begins to act at a certain instant and then moves in some direction with time-dependent speed. The problem considered here is of the third kind.

Representative of the first kind of problem is the steady motion of a line load on the surface of an elastic half-space, which was studied by Sneddon [1] and Cole and Huth [2]. The three-dimensional problem, in which the steady motion of a point force in an unbounded body is studied, was considered by Eason, Fulton and Sneddon [3]. The transient problem of a line load which suddenly appears on the surface of an elastic half-space and then moves off with constant velocity, which is typical of type (ii), was considered by Ang [4] and by Payton [5]. Both employed integral transform methods and a version of the Cagniard-de Hoop [6] technique. The corresponding three-dimensional problem of a pressure-spot suddenly appearing on the surface of an elastic half-space and then moving with constant velocity was considered by Payton [7]. A number of other references to problems of the first and second kinds may be found in the paper by Gakenheimer [8].

* Received August 5, 1971; revised version received October 9, 1971. The support of the National Science Foundation through grant GK26002X to Brown University is gratefully acknowledged. 
The number of available papers treating problems of the third kind is small. Stronge [9] recently presented an exact solution for the complete field in an acoustic half-space due to an accelerating pressure step applied to the surface. The problem of a pressure step decelerating on the surface of an elastic half-space was considered by Friedman, Myers and Bleich [10]. Making use of asymptotic methods, they determined the stress discontinuities at the wave fronts. The response of an elastic half-space to an axisymmetric surface pressure whose region of action expands at a nonuniform rate has been studied by several authors employing various approximate techniques. A list of references may be found in [8]. It seems that no exact solutions for the response of an elastic solid to nonuniformly moving loads have appeared in the literature. Thus, it is the purpose here to present an exact solution for the simplest problem of this type, namely, the nonuniform motion of a line load in an unbounded elastic solid. This problem serves as a convenient vehicle for introducing the analytical method, which may be applied to more complicated problems. Since the purpose here is the discussion of a method, rather than of a problem related to a particular application, only representative calculations are carried out.

Method of solution. Let $x, y, z$ be a Cartesian coordinate system in the body. At time $t=0$ a line load begins to act along the $y$-axis, and the line load moves in the $x$ direction for $t>0$. The magnitude of the line load is such that the force per unit length in the $y$-direction is unity. The line of action may be any direction in the $x, z$-plane. The results for a general line of action, however, may be obtained most easily by superimposing the results for the cases when the line of action is the $z$-direction and when it is the $x$-direction. These two special cases will be called case I and case II, respectively. Because of their similarity, only case I will be treated in detail (see Fig. 1).

For any $t>0$ the $x$-coordinate of the load is given by $l(t)$. For the time being, $l(t)$ is taken to be a continuous, monotonically increasing function of time. Thus, the load occupies neighboring points at neighboring times, and it never acts at a single point for a finite length of time. A discussion of the relaxation of these conditions is deferred to a later point. If the conditions hold, the function $l(t)$ is invertible, that is, there exists
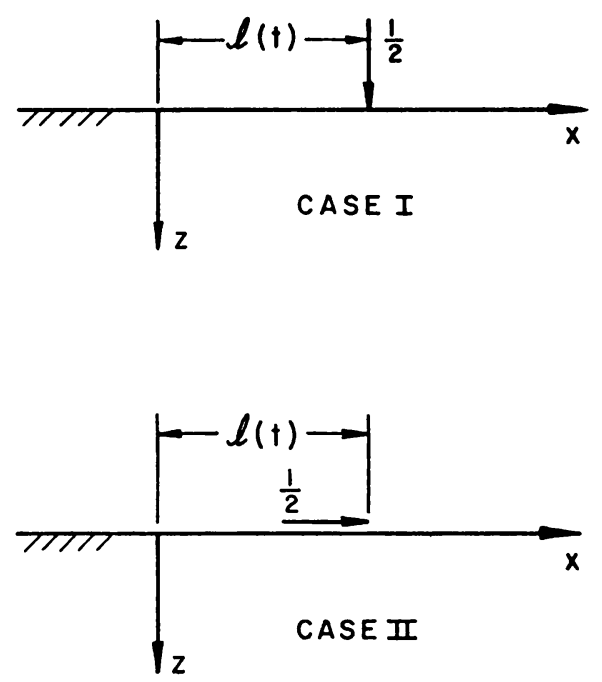

Fig. 1. The $x, z$-plane for the equivalent half-space problems. 
a function $\eta(x)$ which is the time at which the load acts at point $x$. The function $\eta(x)$ is then also continuous and montonically increasing. The time derivative of $l(t)$ is the speed of the load at time $t$, and the spatial derivative of $\eta(x)$ is the slowness of the load at the place $x$. These functions satisfy the following relations identically in $x$ or $t$ :

$$
\begin{aligned}
l[\eta(x)] & =x, & & \eta[l(t)]=t, \\
l[\eta(x)] \eta^{\prime}(x) & =1, & & \eta^{\prime}[l(t)] \dot{l}(t)=1 .
\end{aligned}
$$

Because of their symmetry with respect to the plane $z=0$, both cases I and II may be formulated as boundary value problems for the half-space $z>0$, with mixed boundary conditions on $z=0$. Since the loading is uniform in the $y$-direction, each problem is formulated as one of plane strain. The components of the stress tensor are denoted by $\sigma_{i j}$ and the nonzero components of displacement are $u$ and $w$, all variables being functions of $x, z, t$. If the dilatational displacement potential $\phi$ and the shear displacement potential $\psi$ are introduced, the partial differential equations governing small-amplitude wave motion in the elastic solid are

$$
\phi_{x x}+\phi_{s z}-a^{2} \phi_{t i}=0, \quad \psi_{x z}+\psi_{s z}-b^{2} \psi_{t z}=0
$$

where $a$ and $b$ are the dilatational and shear wave slownesses and subscripts indicate partial derivatives.

The boundary conditions to be satisfied by the solution of (3) are

$$
\begin{array}{ll}
\sigma_{z z}(x, 0, t)=-\frac{1}{2} \delta[x-l(t)], & u(x, 0, t)=0 \\
\sigma_{x z}(x, 0, t)=-\frac{1}{2} \delta[x-l(t)] & w(x, 0, t)=0
\end{array}
$$

where $\delta$ is the Dirac delta function. The factor $\frac{1}{2}$ appears because only one-half of the body is being considered. The formulation is completed by specifying zero initial data. The relation between displacements and the potentials is given in [4], for example, and the stresses and displacements are related by Hooke's law.

The solution of the problem is obtained by making use of Laplace transform methods, interpreting all expressions in their distributional sense. The time is first eliminated by application of the transform

$$
\hat{\phi}(x, z, s)=\int_{0}^{\infty} \phi(x, z, t) e^{-\lrcorner t} d t .
$$

Next, the dependence on $x$ is suppressed by taking the transform

$$
\Phi(\lambda, z, s)=\int_{-\infty}^{\infty} \hat{\phi}(x, z, s) e^{-s \lambda x} d x .
$$

The convention by which the ${ }^{\wedge}$ is used to denote the transform on time and an upper-case letter to denote the transform on $x$ is followed in the subsequent analysis.

Application of the transformed boundary conditions to the bounded solution of the transformed differential equations, taking into account the initial data, yields

$$
\begin{aligned}
& \Phi(\lambda, z, s)=\left(1 / \rho s^{2}\right) A(\lambda, s) \exp (-s \alpha z), \\
& \Psi(\lambda, z, s)=-\left(\lambda / \rho s^{2} \beta\right) A(\lambda, s) \exp (-s \beta z),
\end{aligned}
$$


where $\rho$ is material mass density and

$$
\alpha=\left(a^{2}-\lambda^{2}\right)^{1 / 2}, \quad \beta=\left(b^{2}-\lambda^{2}\right)^{1 / 2} .
$$

The $\lambda$-plane is cut along $a<|\operatorname{Re}(\lambda)|<\infty, \operatorname{Im}(\lambda)=0$ so that $\operatorname{Re}(\alpha) \geq 0$ everywhere in the plane, and similarly for $\beta$. The amplitude $A(\lambda, s)$ in (9) and (10) is essentially the double transform of the inhomogeneous boundary condition, which is easily derived by making use of the relationship (cf. [11, Eq. (53), p. 79])

$$
\delta[x-l(t)]=\eta^{\prime}(x) \delta[t-\eta(x)] .
$$

This relation is strictly valid only if $l(t)$ is continuous and monotonic. In view of (11),

$$
\hat{\sigma}_{z z}(x, 0, s)=-\frac{1}{2} \eta^{\prime}(x) \exp (-s \eta(x)) H[\eta(x)]
$$

for case I. The function $H$ in (12) is the unit step function. Since $\eta(x)$ is a continuous, monotonically increasing function of $x$ and $\eta(0)=0, H[\eta(x)]=H(x)$. Then, applying the two-sided Laplace transform,

$$
\Sigma_{s z}(\lambda, 0, s) \equiv A(\lambda, s)=-\frac{1}{2} \int_{0}^{\infty} \eta^{\prime}(x) e^{-\bullet \lambda x} e^{-\bullet \eta(x)} d x .
$$

The transformed solution is thus completely determined, and the dilatational potential itself may be written as the double inversion integral

$$
\phi(x, z, t)=-\frac{1}{2 \rho} \frac{1}{2 \pi i} \int_{B_{1}} \frac{1}{2 \pi i} \int_{B_{1}} \frac{1}{s} \int_{0}^{\infty} \eta^{\prime}(\xi) e^{(\lambda x-\lambda \xi-\alpha z-\eta(\xi)+t)} d \xi d \lambda d s
$$

where $B_{1}$ and $B_{2}$ are the usual inversion paths for the one-sided and two-sided Laplace transforms. The double integral in (14) is inverted formally by means of de Hoop's technique [6], after which it can be verified that the result does indeed satisfy the differential equation (3).

The first step in the evaluation is an interchange of order of integration to yield

$$
\phi(x, z, t)=-\frac{1}{2 \rho} \int_{0}^{\infty} \eta^{\prime}(\xi) \frac{1}{2 \pi i} \int_{B_{2}} \frac{1}{2 \pi i} \int_{B_{2}} \frac{1}{s} e^{*()} d \lambda d s d \xi
$$

where the exponent is the same as in (14). The next step is to transform the path of integration $B_{2}$ into another path such that the inversion integral along $B_{1}$ may be evaluated by observation. To this end, a path is sought along which

$$
\begin{aligned}
& \operatorname{Re}(-\lambda x+\lambda \xi+\alpha z)=\tau \geq 0, \\
& \operatorname{Im}(-\lambda x+\lambda \xi+\alpha z)=0 .
\end{aligned}
$$

Such a path is given by

$$
\lambda=\lambda_{ \pm}(x-\xi, z, \tau)=r^{-2}\left[-\tau(x-\xi) \pm i\left(\tau^{2}-r^{2} a^{2}\right)^{1 / 2} z\right],
$$

which defines one branch of a hyperbola with vertex at $\lambda=a(\xi-x) / r$ and asymptotes $\arg (\lambda)= \pm z /(x-\xi)$. The distance $r$ in (17) is defined by $\left[(x-\xi)^{2}+z^{2}\right]^{1 / 2}$. The parameter $\tau$ varies from $r a$ to $\infty$ as $\lambda$ moves away from the real axis in either the upper or lower half-plane. Furthermore, the hyperbola does not intersect any branch cuts of the integrand. Cauchy's theorem is then applied to show that the integral along $B_{2}$ equals the integral along the hyperbolic path, which may be converted to a real integral to yield 


$$
\phi=-\frac{1}{2 \rho} \int_{0}^{\infty} \frac{\eta^{\prime}(\xi)}{2 \pi i} \int_{B_{1}} \frac{1}{\pi s} \int_{r a}^{\infty} \operatorname{Im}\left[\frac{\partial \lambda_{+}}{\partial \tau}\right] e^{s(t-\eta(\xi)-r)} d \tau d s d \xi .
$$

Next, the order of integration over $\tau$ and $s$ is interchanged, and the integral over $s$ is recognized as the unit step function $H[t-\eta(\xi)-\tau a]$. Consequently, (18) may be rewritten

$$
\phi=-\frac{1}{2 \pi \rho} \int_{L} \eta^{\prime}(\xi) \int_{r 0}^{t-\eta(\xi)} \operatorname{Im}\left[\frac{\partial \lambda_{+}}{\partial \tau}\right] d \tau d \xi
$$

where $L=L(x, z, t)$ is the union of subintervals of $0 \leq \xi<\infty$ on which $t-\eta(\xi)-$ $r a>0$. Finally, if the explicit expression for the integrand is substituted into (19), the integral may be easily evaluated to yield

$$
\phi(x, z, t)=-\frac{1}{2 \pi \rho} \int_{L} \eta^{\prime}(\xi) z r^{-2}\left\{[t-\eta(\xi)]^{2}-r^{2} a^{2}\right\}^{1 / 2} d \xi .
$$

The expression for $\psi(x, z, t)$ is the same, except that $z$ and $x-\xi$ are interchanged in the integrand and $a$ is replaced by $b$. It can be shown that the derived result (20) is indeed a solution of the initial-boundary value problem, at least in the sense of distributions [12].

The solution for case II is the same as for case I, except that $(x-\xi)$ and $z$ are interchanged in $\phi(x, z, t)$ and $(x-\xi)$ and $-z$ are interchanged in $\psi(x, z, t)$.

At this point it is a simple matter to relax the condition that $l(t)$ must be monotonically increasing. By making a change of variable from arc length along the load trajectory to elapsed time $\tau$ in (20), the solution takes the form

$$
\phi(x, z, t)=-\frac{1}{2 \pi \rho} \int_{N} z r^{-2}\left[(t-\tau)^{2}-r^{2} a^{2}\right]^{1 / 2} d \tau
$$

where $r=\left\{[x-l(\tau)]^{2}+z^{2}\right\}^{1 / 2}$ and $N=N(x, z, t)$ is the union of subintervals of $0 \leq$ $\tau<\infty$ on which $t-\tau-r a \geq 0$. It can be verified by direct substitution that this form of the result also satisfies the wave equation and boundary conditions for quite general functions $l(t)$. For example, an oscillating load whose position is described by $l(t)=\sin \omega t$ may be considered. For most applications, however, it seems that the requirement that $l(t)$ be monotonic is not a hindrance.

Discussion of the solution. A useful viewpoint to take in studying moving-load problems is to think of the force as being a source of elementary signals or wavelets at each point of its trajectory. For the problem at hand, the load generates cylindrical dilatational and shear wavelets from each point of its trajectory along the $x$-axis. Typical wavelets are emitted from the point $(\xi, 0)$ at time $\eta(\xi)$, and thereafter propagate outward with slownesses $a$ and $b$. The radius at some later time $t$ of the dilatational wavelet is $[t-\eta(\xi)] / a$. The time at which a particular wavelet reaches a field point $(x, z)$ is the sum of the time at which it was emitted, $\eta(\xi)$, plus the travel time from the source point $(\xi, 0)$ to the field point, which is just $r a$. The time of arrival is then $\eta(\xi)+r a$. Therefore, for any particular time $t$ and place $(x, z)$, those points on the trajectory $0 \leq \xi<\infty$ for which $t>\eta(\xi)+r a$ contribute to the solution, while the motion of the load along those points for which $t<\eta(\xi)+r a$ is undetected. This is, of course, exactly the criterion used to determine the intervals of integration $L$ in the solution (20).

The wavefronts, which may be defined as curves along which discontinuities or 
algebraic infinities of the solution may exist, are expected to be more complicated in the case of nonuniform load motion than in the case of uniform motion. If the load suddenly appears at $(0,0)$ and then moves off at constant speed along the $x$-axis, circular wavefronts emanating from $(0,0)$ will appear. Furthermore, if the load speed is subsonic these will be the only fronts, while if the load is supersonic with respect to either or both wave speeds, straight-fronted Mach waves trail the moving load. In the case of a nonuniformly moving load the Mach waves take on more complicated shapes, but these can be determined explicitly by the method of characteristics. As in the case of constant-velocity loads, the circles centered at $(0,0)$ of radius $t / a$ and $t / b$ must be admitted as possible wavefronts. Only the Mach waves will receive special attention here.

The wavefronts are essentially the intersection of a plane $t=$ constant with the characteristic surfaces of the governing differential equations. For the problem at hand, these are the real surfaces which satisfy the eikonal equation for either wave speed and which contain the trajectory of the point of application of the load in $x, z, t$-space [13]. Since the determination of the shear wavefronts differs only in minor details from determination of the dilatational wavefronts, only the latter will be considered here.

Suppose the equation of a characteristic has the form

$$
t=\chi(x, z),
$$

where $\chi$ solves the equation

$$
\chi_{x}^{2}+\chi_{z}^{2}=a^{2} .
$$

As shown in [13], for example, the characteristic surface may be found by integration of the system of five ordinary differential equations

$$
\frac{d x}{d \nu}=p, \quad \frac{d p}{d \nu}=0, \quad \frac{d t}{d \nu}=0, \quad \frac{d z}{d \nu}=q, \quad \frac{d q}{d \nu}=0
$$

where $p=\chi_{2}, q=\chi_{1}$, and $\nu$ is a measure of distance along a ray. Each ray has a point in common with the load trajectory and this point is chosen as $\nu=0$. The system (24) is subjected to the initial conditions that, at $\nu=0$,

$$
x=x_{0}=l\left(t_{0}\right), \quad p=p_{0}, \quad t=t_{0}, \quad z=z_{0}=0, \quad q=q_{0}
$$

where $t_{0}$ is a real positive parameter called the "initial" time. The initial data must be restricted by the condition that $t_{0}=\chi\left(x_{0}, z_{0}\right)$, which is most useful in its local form

$$
1=p_{0} i\left(t_{0}\right) \text {. }
$$

Furthermore, the initial data must be compatible with the eikonal equation (23),

$$
p_{0}^{2}+q_{0}^{2}=a^{2} .
$$

Under these conditions, the unique solution of (24) may be written in the form, upon elimination of $\nu$,

$$
\begin{aligned}
& x=\left(\left(t-t_{0}\right) / i\left(t_{0}\right) a^{2}\right)+l\left(t_{0}\right), \\
& z=\left(\left(t-t_{0}\right) / a^{2}\right)\left(a^{2}-\left(1 / i_{0}^{2}\right)\right)^{1 / 2} .
\end{aligned}
$$

Eqs. (28) describe the wavefront at time $t$ parametrically in $t_{0}$. If $t_{0}$ were eliminated and the resulting equation solved for $t$ as a function of $x$ and $z$, this function would be 
$\chi(x, z)$. For arbitrary $l(t)$, of course, these steps cannot be carried out. The wavefronts may alternately be described in terms of spatial coordinate $\xi$ along the load trajectory, rather than initial time. This may be done by replacing $l\left(t_{0}\right), t_{0}$ and $i\left(t_{0}\right)$ in (28) by $\xi, \eta(\xi)$ and $1 / \eta^{\prime}(\xi)$, respectively. Eqs. (28) may also be derived by viewing the wavefronts as envelopes of elementary wavelets [12].

As can be seen from (28), a necessary condition for the existence of a wavefront is that $i\left(t_{0}\right) a \geq 1$. This implies that the load must be moving supersonically at $t_{0}$ with respect to the dilatational wave speed in order to generate a dilatational wavefront. When $i(t)$ is constant, the wavefronts are clearly straight lines. The wavefronts for two special cases of nonuniform motion are shown in Fig. 2. In Fig. 2a, $l=c_{1} t^{2}$ and in Fig. 2b, $l=c_{2} t^{1 / 2}$, where $c_{1}$ and $c_{2}$ are constants. For ease of calculation the units have been adjusted so that $a$ has magnitude one, $c_{1}$ has magnitude one and $c_{2}$ has magnitude two. The inverse function for $l=t^{2}$ is given by $\eta=\xi^{1 / 2}$, and for $l=2 t^{1 / 2}$ it is given by $\eta=\xi^{2} / 4$. The solid lines in the figures represent dilatational wavefronts and the dashed lines represent shear wavefronts. All calculations were carried out for Poisson's ratio 0.25.

In the first example, where $l=t^{2}$, the load accelerates from zero velocity through the sonic speeds of the material. The time at which the speed of the load is the dilatational wave speed is $t=\frac{1}{2}$, and the $x$-coordinate of the load at this time is $\frac{1}{4}$. The wavefronts are shown in Fig. 2a for time $t=2$. At this time the load speed has accelerated to four times the dilatational wave speed. The dilatational Mach wave trailing the load was determined by setting $t=2$ in (28), and letting $t_{0}$ range from $\frac{1}{2}$ to 2 to obtain the $x$ and $z$ coordinates of the front. The result is the entire curve $A B C$ in Fig. 2a. This curve is the envelope of elementary wavelets emitted between times $t=\frac{1}{2}$ and $t=2$, the cusp at $B$ appearing naturally as a result of the calculation. As pointed out by Stronge [9]; the segment $B C$ is not a portion of an elementary wavelet centered at the point at which the load passes through the sonic speed. Rather, the point $C$ lies on the wavelet centered at $\left(\frac{1}{4}, 0\right)$ and the rest of the segment $B C$ lies behind this wavelet. Contrary to the impli-

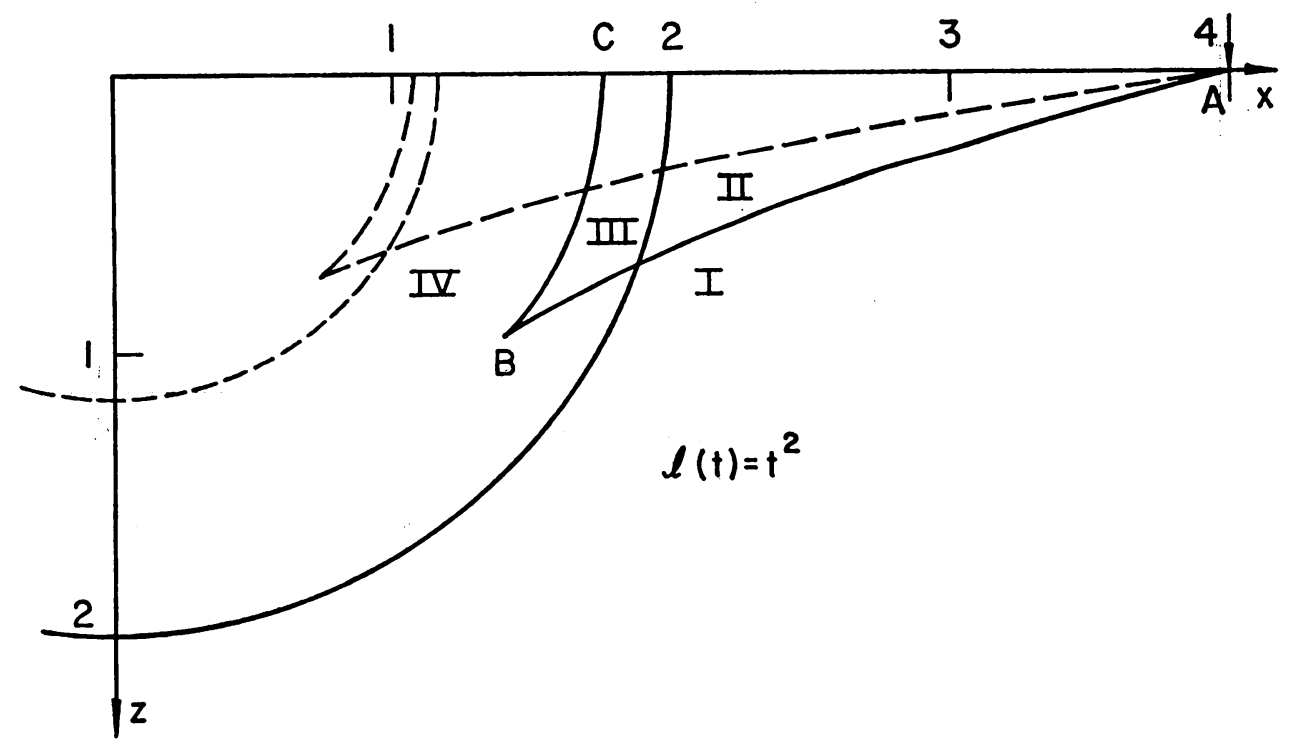

Fig. 2a. The wavefronts for the particular case of an accelerating load. 


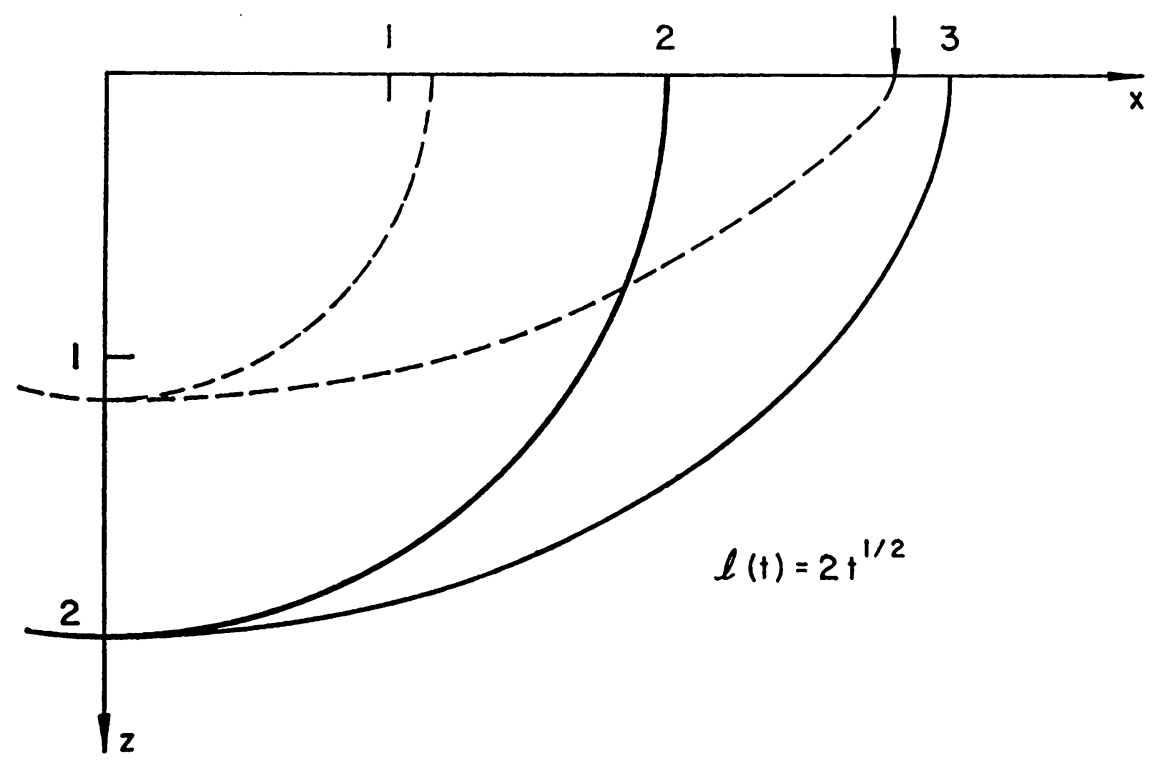

Fig. 2b. The wavefronts for the particular case of a decelerating load.

cation made by Stronge [9], however, the entire curve $A B C$ is an envelope of wavelets. Similar remarks apply for the shear wavefronts.

Referring to Fig. 2a, the $x, z$-plane is divided into four regions for $t=2$, indicated by Roman numerals. The boundaries of the regions are the dilatational wavefronts (the solid lines). It is interesting to plot $T \equiv t-\eta(\xi)-r a$ versus $\xi$ for typical points in each of these regions to see which segments of the load trajectory contribute to the solution at that point. It is sufficient to consider the portion of the trajectory $0 \leq \xi \leq 4$. The results are shown in Fig. 3. In region I the load is undetected. In II, the dilation at a

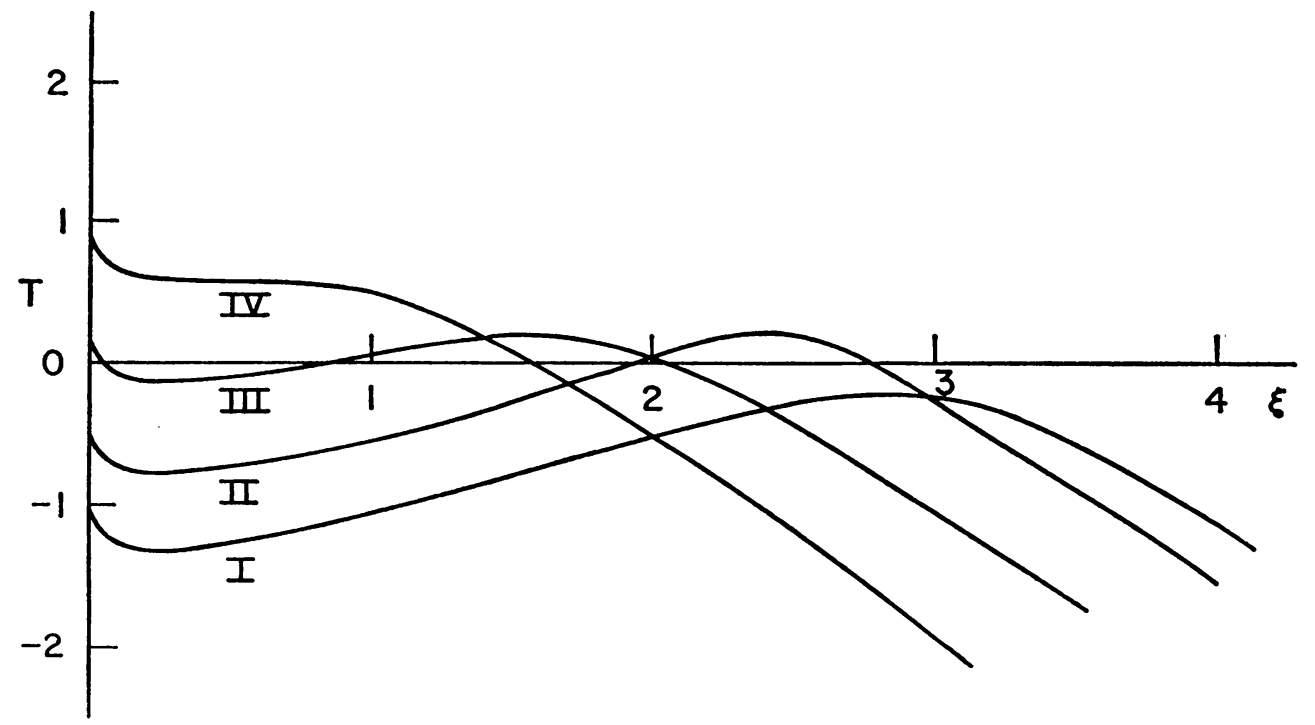

Fra. 3. A plot of $t-r a-\eta(\xi)$ versus $\xi$ for the case shown in Fig. 2a. 
typical point depends on the motion of the load along a part of the path on which the load moves supersonically. The same is true in III, with the addition that the dilation also depends on a segment of the trajectory extending outward from $\xi=0$ on which the load moves subsonically. In IV, $\phi$ depends only on a segment extending from $\xi=0$. The dependence of $\psi$ on load trajectory is similar.

Fig. $2 \mathrm{~b}$ shows the wavefronts for the case of a load decelerating from an initially very large speed through the wave speeds of the material, that is, $l(t)=2 t^{1 / 2}$. The load decelerated through the dilatational wave speed at $t=1$, and the wavefronts are shown for $t=2$. At this time, the load is still moving supersonically with respect to the shear wave speeds. As can be seen in the figure, the dilatational front has separated from the load while the shear front still trails the moving load. Separation of a front from the load occurs when the load decelerates through a wave speed. Although the details are quite different from the accelerating load case, the general features are the same. Many of the details for a load decelerating from a finite initial velocity are worked out in [10].

Asymptotic wavefront analysis. In this section a representative calculation is carried out to determine the strength of the mean normal stress near the wavefronts. The details of such a calculation depend a great deal on the character of the function $l(t)$, that is, on whether the load is accelerating or decelerating, for example. If the existence of wavefronts is assumed from the outset, however, a general asymptotic result which is applicable in most cases may be derived. The derivation is based on the method developed by Knopoff and Gilbert [14] for extracting first motion solutions from the complete transformed solution. The Laplace transform on time of the mean normal stress is proportional to $s^{2} \hat{\phi}$, the proportionality factor being $\kappa a^{2}$, where $\kappa$ is the bulk modulus.

From (8),

$$
\hat{\phi}(x, z, s)=\frac{-1}{2 \rho s} \int_{0}^{\infty} \eta^{\prime}(\xi) \frac{1}{2 \pi i} \int_{B_{z}} e^{\cdot(\lambda z-\lambda \xi-\alpha z-\eta)} d \lambda d \xi .
$$

The main step in the analysis is the approximation of this integral for large $s$. As discussed by Knopoff and Gilbert [14], both positive and negative values of $s$ must be considered. For most field points $(x, z)$ the main contribution to the integral comes from a neighborhood of any point $\left(\lambda_{0}, \xi_{0}\right)$ satisfying $\partial h / \partial \lambda=0$ and $\partial h / \partial \xi=0$ [15], where

$$
h(\lambda, \xi) \equiv \lambda x-\lambda \xi-\alpha z-\eta \text {. }
$$

Letting $\xi_{0}$ be a root of

$$
\eta^{\prime}(\xi)-a(x-\xi) / r=0,
$$

assuming it has a root, it can be shown that

$$
\lambda_{0}=-a\left(x-\xi_{0}\right) / r_{0}
$$

where $r_{0}$ is just $r$ evaluated at $\xi=\xi_{0}$. In view of (31) and (32) it can be seen that the path of integration $B_{2}$ in (29) does not include the point $\lambda_{0}$. However, a transformation of the path to the same path (17) used in the exact inversion procedure results in the stationary point being on the integration contour. Then by paralleling the steps taken in going from (15) to (18), it is found that

$$
\dot{\phi}=\frac{-1}{2 \rho s} \int_{0}^{\infty} \eta^{\prime}(\xi) \int_{0}^{\infty} \frac{1}{\pi} \operatorname{Im}\left[\frac{\partial \lambda_{ \pm}}{\partial \tau}(x-\xi, z, r a+\gamma)\right] e^{-a \gamma} d \gamma e^{-\cdot(r a+\eta)} d \xi .
$$


The inner integral represents the contribution from individual wavelets. Only the first motions of the elementary wavelets are important near the wavefronts, so the inner integral is evaluated approximately by Laplace's method as $s \rightarrow+\infty$. The main contribution to the integral comes from the vicinity of $\gamma=0$ where the integrand is singular of order one-half. A straightforward application of Watson's lemma [15], however, yields

$$
\hat{\phi} \simeq \frac{-z a^{1 / 2}}{2^{3 / 2} \rho s^{3 / 2}} \int_{0}^{\infty} \eta^{\prime}(\xi) r^{-3 / 2} e^{-\imath(r a+\eta)} d \xi .
$$

The main contributions to the integral in (34) come from near the end point $\xi=0$ and from near the roots of $d g / d \xi=0$, where

$$
g(\xi) \equiv \eta(\xi)+r a .
$$

As before, a typical root will be denoted by $\xi_{0}$. The contribution from near $\xi=0$ represents the solution just behind the circular wavefront centered at $(0,0)$. This may be derived in the manner outlined in [14] .Only the contribution from near the stationary points of $g$ will receive further attention here.

Expanding $g(\xi)$ in a Taylor series about the point $\xi=\xi_{0}$, we find that the main contribution to the integral from this point for large $|s|$ takes the form [14]

$$
\hat{\phi} \simeq \frac{-z e^{-0 \sigma_{0}} O a^{1 / 2}}{2^{3 / 2} \rho s^{3 / 2}} \int_{\xi_{0}-R}^{\xi_{0}+R} \eta^{\prime}(\xi) r^{-3 / 2} e^{-1 / 20_{0}{ }^{\prime \prime}\left(\xi-\xi_{0}\right) 2} d \xi
$$

where $R$ is a positive real number.

If $g_{0}^{\prime \prime}>0$ then the stationary point is a minimum, the asymptotic value of the integral is determined as $s \rightarrow+\infty$, and the result represents the solution just behind the corresponding wavefront. That is, the wavefront arrival at $(x, z)$ marks the onset of a certain type of motion. On the other hand, if $g_{0}^{\prime \prime}<0$ then the stationary point is a maximum, the asymptotic value of the integral is determined as $s \rightarrow-\infty$, and the result represents the solution just in front of the corresponding wavefront. In this case, the wavefront arrival marks the termination of a certain type of wave motion. (In many problems in elastic wave propagation, a particular shear wavefront marks both the onset and termination of certain wave motions, giving rise to the terminology "two-sided shear wave" [14].) In either case, the value of the integral in (36) is essentially unaffected by letting $R \rightarrow \infty$ if $|s|$ is sufficiently large. The result of the approximate evaluation of (36) is then

$$
\hat{\phi}_{0} \simeq \frac{-z a^{1 / 2} \eta_{0}^{\prime} \exp \left(-s g_{0}\right)}{2 \rho s^{2} r_{0}\left|\eta_{0}^{\prime \prime}\right|^{1 / 2}}\left|\frac{\eta_{0}^{\prime \prime}}{r_{0} g_{0}^{\prime \prime}}\right|^{1 / 2} .
$$

This asymptotic result is valid for all points except possibly near those points at which the wavefront has a cusp. Such points appear to correspond to inflection points of the curve $g(\xi)=0$, and the asymptotic analysis must be modified accordingly [15].

The form of (37) indicates that, at a particular point $(x, z)$, a wavefront arrives at time $t=g_{0}$. Furthermore, it can be verified by a long but straightforward calculation that $\left|\eta_{0}^{\prime \prime} / r_{0} g_{0}^{\prime \prime}\right|$ is exactly the curvature of the wavefront at $(x, z)$ at time $t=g_{0}$. The curvature factor yields the familiar behavior for two-dimensional problems that the wavefront amplitude depends on the square root of the curvature. The intensity of the solution at the front increases or decreases according as the wavefront is concave or convex toward the direction of propagation. The example illustrated in Fig. $2 \mathrm{a}$ shows 
that both cases may occur in the same problem. It can be verified directly from (37) that the decay factor for the case of a constant velocity load is unity.

Referring again to the particular case of $l(t)=t^{2}$ shown in Fig. 2a, the function $g(\xi)$ has one local maximum and one local minimum in $0<\xi<\infty$ for any point $(x, z)$ in the path of the Mach wave. The minimum corresponds to the front $A B$ and the maximum corresponds to $B C$. Near the cusp at $B$ the minimum and maximum are very close, finally coalescing at $B$. This corresponds to an inflection point of $g(\xi)$, and the analysis requires modification. On the other hand, for any $(x, z)$ not in the path of the Mach waves the function $g(\xi)$ has no stationary points and its minimum occurs at $\xi=0$, corresponding to the circular wavefront. Similar remarks may be made for the case $l=2 t^{1 / 2}$.

\section{REFERENCES}

[1] I. N. Sneddon, Fourier transforms, McGraw-Hill, New York, 1951, p. 445

[2] J. D. Cole and J. Huth, J. Appl. Mech. 25, 433 (1958)

[3] G. Eason, J. Fulton and I. Sneddon, Phil. Trans. Roy. Soc. A248, 575 (1956)

[4] D. D. Ang, Quart. Appl. Math. 18, 251 (1960)

[5] R. G. Payton, Int. J. Engng. Sci. 5, 49 (1967)

[6] A. T. deHoop, Appl. Sci. Res. B8, 349 (1960)

[7] R. G. Payton, Quart Appl. Math. 21, 299 (1964)

[8] D. C. Gakenheimer, J. Appl. Mech. 38, 99 (1971)

[9] W. J. Stronge, J. Appl. Mech., 37, 1077 (1970)

[10] M. B. Friedman, M. K. Myers and H. H. Bleich, Tech. Report DASA 2144, 1968

[11] B. van der Pol and H. Bremmer, Operational calculus, Cambridge, 1964

[12] L. B. Freund, Brown University Report, GK-26002X/1, 1971

[13] F. G. Friedlander, Sound pulses, Cambridge, 1958

[14] L. Knopoff and F. Gilbert, J. Acoust. Soc. Am. 31, 1161 (1959)

[15] L. Sirovich, Techniques of asymptotic analysis, New York, Springer-Verlag, 1971 